\title{
Influence of weight functions to a nonlocal $p$-Laplacian evolution equation with inner absorption and nonlocal boundary condition
}

\section{Zhong Bo Fang ${ }^{*}$ and Jianyun Zhang}

"Correspondence:

fangzb7777@hotmail.com School of Mathematical Sciences, Ocean University of China, Qingdao, 266100, P.R. China

\begin{abstract}
In this paper, we investigate an initial boundary value problem for a nonlocal $p$-Laplacian evolution equation with an inner absorption and weighted linear nonlocal boundary and initial conditions. By using the modified comparison principle and the method of upper-lower solution, we find the influence of weighted function on determining blow-up or not of nonnegative solutions.
\end{abstract}

\section{Introduction}

We consider a nonlocal $p$-Laplacian evolution equation with an inner absorption term

$$
u_{t}-\operatorname{div}\left(|\nabla u|^{p-2} \nabla u\right)=u^{m} \int_{\Omega} u^{n}(y, t) d y-\alpha u^{r}, \quad(x, t) \in \Omega \times(0,+\infty),
$$

subject to the weighted linear nonlocal boundary and initial conditions

$$
\begin{aligned}
& u(x, t)=\int_{\Omega} \varphi(x, y) u(y, t) d y, \quad(x, t) \in \partial \Omega \times(0,+\infty), \\
& u(x, 0)=u_{0}(x) \geq 0, \quad x \in \bar{\Omega},
\end{aligned}
$$

where $p>2, m \geq 0, n>0, r \geq 1, \alpha>0$, and $\Omega \subset R^{N}(N \geq 1)$ is a bounded domain with smooth boundary. The weight function $\varphi(x, y) \not \equiv 0$ in the boundary condition is nonnegative and continuous on $\partial \Omega \times \bar{\Omega}$, and $\int_{\Omega} \varphi(x, y) d y>0$ on $\partial \Omega$. The initial function $u_{0}(x) \in C(\bar{\Omega}) \cap W_{0}^{1, p}(\Omega)$ is a nonnegative function satisfying the compatibility condition and $\frac{\partial u_{0}(x)}{\partial v}<0$, where $v$ denotes the unit outer normal vector on the boundary.

Many natural phenomena, such as the non-Newton flux in the mechanics of fluid, population of biological species, and filtration, have been formulated as nonlocal diffusive equation (1.1); see $[1,2]$ and references therein. In the diffusion system of some biological species with human-controlled distribution, $u(x, t), \operatorname{div}\left(|\nabla u|^{p-2} \nabla u\right), \int_{\Omega} u^{m}(y, t) d y$, and $-\alpha$ represent the density of the species at location $x$ and time $t$, the mutation, the humancontrolled distribution, and the decrement rate of biological species, respectively. The evolution of the species at a point of space depends not only on the density of species in a partial region but also in the total region because of the nonlocal boundary condition that arises from the spatial inhomogeneity $(c f$. [3-5]). In fact, there are some important 
phenomena formulated as parabolic equations which are coupled with weighted nonlocal boundary conditions in mathematical models such as thermoelasticity theory. In this case, the solution $u(x, t)$ describes entropy per volume of the materia1 $(c f .[6,7])$.

In the last decades, there have been many researchers dealing with blow-up solutions to the initial boundary value problems of equations with or without nonlocal boundary conditions; see [8-16] and references therein. For the studies of the initial boundary value problem for a local parabolic equation with weighted nonlocal boundary condition (1.2), we refer to [8-10]. For example, Friedman [8] and Pao [9] considered the initial boundary value problem of linear and semi-linear parabolic equations, respectively. They studied the asymptotic behavior of solutions and found some effects of a weight function on blow-up and global solutions. Wang et al. [10] studied the porous medium equation with terms of power form

$$
u_{t}=\Delta u^{m}+u^{p}, \quad(x, t) \in \Omega \times(0,+\infty) .
$$

By virtue of the upper-lower solution method, they obtained the existence of global solutions, blow-up properties and blow-up rate of the solutions.

For the study of the initial boundary value problem of a nonlocal parabolic equation with weighted nonlocal boundary condition (1.2), we refer to [11, 12,15]. For example, Lin et al. [11] considered the semi-linear parabolic equation

$$
u_{t}=\Delta u+\int_{\Omega} g(u) d x, \quad(x, t) \in \Omega \times(0,+\infty)
$$

with nonlocal boundary condition (1.2). They established the existence of local and global solutions and blow-up properties of the solutions. Moveover, they derived the uniform blow-up estimates for a special $g(u)$ under suitable assumptions. Yang et al. [12] discussed the nonlocal slow diffusion equation

$$
u_{t}=\Delta u^{m}+a u^{p} \int_{\Omega} u^{q}(y, t) d y, \quad(x, t) \in \Omega \times(0,+\infty),
$$

and they showed the existence of global solutions, blow-up properties, and blow-up rates of the solutions. For the Dirichlet initial boundary problem of nonlocal evolutionary equation, see $[13,14]$ and references therein for details.

Recently, Wang et al. [15] studied the following semi-linear parabolic equation with nonlocal source and interior absorption terms:

$$
u_{t}=\Delta u+\int_{\Omega} u^{m} d x-\alpha u^{r}, \quad(x, t) \in \Omega \times(0,+\infty)
$$

with weighted linear nonlocal boundary condition (1.2) and initial condition (1.3), where $m, r \geq 1$ and $\alpha>0$. By using the comparison principle and the technique of upper-lower solutions, they obtained the following results:

- If $1 \leq m<r$, then the global solution of the problem exists.

- If $m>r \geq 1$, the problem has solutions that blow up in finite time as well as global solutions, i.e.,

(i) if $\int_{\Omega} \varphi(x, y) d y \leq 1$ and $u_{0}(x) \leq\left(\frac{\alpha}{|\Omega|}\right) \frac{1}{r-m}$, then the global solution exists; 
(ii) if $\int_{\Omega} \varphi(x, y) d y>1$ and $u_{0}(x)>\left(\frac{\alpha}{|\Omega|-\alpha}\right)^{\frac{1}{r}},(|\Omega|>\alpha)$, then the solution blows up in finite time;

(iii) for any $\varphi(x, y) \geq 0$, there exists $a_{1}>0$ such that the solution blows up in finite time provided that $u_{0}(x)>a_{1} \phi(x)$, where $\phi(x)$ is the corresponding normalized eigenfunction of $-\triangle$ with the homogeneous Dirichlet boundary condition and $\int_{\Omega} \phi(x) d x=1$.

- If $m=r>1$, then

(i) the solution blows up in finite time for any $\varphi(x, y) \geq 0$ and large enough $u_{0}$;

(ii) for some positive constant $a_{2}$, the global solution exists for any $u_{0}(x) \leq a_{2} \psi(x)$ provided that $\int_{\Omega} \varphi(x, y) d y<1$, where $\psi(x)$ is a function that solves the following problem:

$$
\begin{aligned}
& -\triangle \psi(x)=\delta_{0}, \quad x \in \Omega, \\
& \psi(x)=\int_{\Omega} \psi(x, y) d y, \quad x \in \partial \Omega .
\end{aligned}
$$

Here, $\delta_{0}$ is a positive constant such that $0 \leq \psi(x) \leq 1$.

In addition, for the initial boundary value problem of equation (1.4) with the homogeneous Dirichlet boundary condition, we refer to [16] and references therein.

We know from the works mentioned above that the studies of problem (1.1)-(1.3) have not been developed well. The difficulty lies in finding the influence of a weight function in the boundary condition and the competitive relationship between nonlocal source and inner absorption to determine blow-up or not of solutions. Motivated by it, we intend to find the sufficient conditions of the existence of global and blow-up solutions of problem (1.1)-(1.3) by using the method of upper-lower solution, the technique of ordinary differential equation, the method of characteristic function and self-similar lower solution. Here, the key is to establish a modified comparison principle. We will use the suitable test function and Gronwall's inequality to derive it. Besides, the main contribution of this paper is our results including the nonlocal nonlinearity Hölder (non-Lipschitz) cases for $m$ or $n \in(0,1)$, as well as the Lipschitz cases for $m, n \geq 1$, and covering all the results of $[11,15]$. Our detailed results are as follows.

Theorem 1 Suppose that $m+n>r \geq 1$. If $\int_{\Omega} \varphi(x, y) d y \geq 1$ for $x \in \partial \Omega$ and $u_{0}(x)>\left(\frac{\alpha}{|\Omega|-\alpha}\right)^{\frac{1}{m}}$ $(|\Omega|>\alpha)$, then the solution of problem (1.1)-(1.3) blows up in finite time.

Theorem 2 Suppose that $m+n<\max \{p-1, r\}$. If $\int_{\Omega} \varphi(x, y) d y \leq \frac{1}{a}<1$ for $x \in \partial \Omega$, then the global solution of problem (1.1)-(1.3) exists for small initial data.

Theorem 3 Suppose that $m+n>\max \{p-1, r\}$. If $\int_{\Omega} \varphi(x, y) d y \leq \frac{1}{a}<1$ for $x \in \partial \Omega$, then the negative global solution of problem (1.1)-(1.3) exists for small initial data, and the nonnegative solution blows up in finite time for arbitrary $\varphi(x, y)>0$ and sufficiently large initial data.

Theorem 4 Suppose that $m+n=\max \{p-1, r\}$.

(i) If $\int_{\Omega} \varphi(x, y) d y \leq 1, r>p-1$, and $\alpha \geq|\Omega|$, then the global solution of problem (1.1)-(1.3) exists for small enough initial data, and if $\psi^{m} \int_{\Omega} \psi^{n} d x>\alpha \psi^{r}$ and 
$\int_{\Omega} \varphi(x, y) d y \geq a>1$, then the solution of problem (1.1)-(1.3) blows up in finite time for sufficiently large initial data.

(ii) If $\psi^{m} \int_{\Omega} \psi^{n} d x \leq 1, \int_{\Omega} \varphi(x, y) d y \leq \frac{1}{a}<1$, and $p-1>r$, then there exists a global solution of problem (1.1)-(1.3) for small enough initial data, and if $\psi^{m} \int_{\Omega} \psi^{n} d x>1$ and $\int_{\Omega} \varphi(x, y) d y \geq a>1$, then the solution of problem (1.1)-(1.3) blows up in finite time for sufficiently large initial data.

Remark 1 It is easy to see that there is no blow-up solution for $m=r=1$ in Theorem 4 .

Remark 2 The exact values of sufficiently large or small initial data stated in the theorems above will be given in the proofs of Section 3.

Remark 3 The function $\psi(x)$ solves problem (3.2)-(3.3) and $a$ is a constant defined in (3.4).

The rest of our paper is organized as follows. In Section 2, with the definitions of weak upper and lower solutions, we give the modified comparison principle for problem (1.1)(1.3), which is an important tool for our research. The proofs of our main results are given in Section 3.

\section{Comparison principle and local existence}

Since equation (1.1) is degenerate when $p>2$, there is no classical solution in general. Hence, it is reasonable to find a weak solution of (1.1). To this end, we first give the following definitions of lower and upper nonnegative weak solutions of problem (1.1)-(1.3). Let $Q_{T}=\Omega \times(0, T), S_{T}=\partial \Omega \times(0, T)$, and let $\bar{\Omega}_{T}=\Omega \times[0, T)$.

Definition 1 If a nonnegative function $\underline{u}(x, t)$ satisfies the following conditions:

(1) $\underline{u} \in C\left(0, T ; L^{\infty}(\Omega)\right) \cap L^{p}\left(0, T ; W_{0}^{1, p}(\Omega)\right), \underline{u}_{t} \in L^{2}\left(0, T ; L^{2}(\Omega)\right), \underline{u}(x, 0) \leq \underline{u}_{0}(x), x \in \bar{\Omega}_{T}$,

(2) $\iint_{Q_{T}} \underline{u}_{t} \phi d x d t+\iint_{Q_{T}}|\nabla \underline{u}|^{p-2} \nabla \underline{u} \cdot \nabla \phi d x d t \leq \iint_{Q_{T}} \phi\left(\underline{u}^{m} \int_{\Omega} \underline{u}^{n}(y, t) d y-\right.$ $\left.\alpha \underline{u}^{r}\right) d x d t$, where $\phi \in \Theta=\left\{\chi: \chi \in C^{1,1}(\bar{\Omega}) ; \chi(x, t) \geq 0 ; \chi(x, T)=0 ; \chi(x, t)=0\right.$ on $\left.S_{T}\right\}$,

(3) $\underline{u}(x, t) \leq \int_{\Omega} \varphi(x, y) \underline{u}(y, t) d y, x \in S_{T}$,

then $\underline{u}(x, t)$ is called a weak lower solution of problem (1.1)-(1.3).

Similarly, we can define the weak upper solution $\bar{u}(x, t)$ of problem (1.1)-(1.3). We say that $u(x, t)$ is a weak solution of problem (1.1)-(1.3) in $Q_{T}$ if it is both weak lower and upper solution of problem (1.1)-(1.3) in $Q_{T}$.

Lemma 1 Suppose that $\xi$ and $\bar{\xi}$ are in $R^{N}$. If $p \geq 2$, then there exists a positive constant $c>0$ such that

$$
\left(|\bar{\xi}|^{p-2} \bar{\xi}-|\xi|^{p-2} \xi\right) \cdot(\bar{\xi}-\xi) \geq c|\bar{\xi}-\xi|^{p}, \quad \text { where } c=c(N, p) \text { depends on only } N \text { and } p
$$

Remark 4 Lemma 1 can be shown in a similar manner as the one used in Lemma 4.10 in [17].

The following modified comparison principle plays a crucial role in our proofs, which can be obtained by establishing a suitable test function and using Lemma 1 and Gronwall's inequality. 
Proposition 1 (Comparison principle) Suppose that $\underline{u}(x, t)$ and $\bar{u}(x, t)$ are nonnegative weak lower and upper solutions of problem (1.1)-(1.3), respectively, with $\underline{u}(x, 0) \geq 0$, $\bar{u}(x, 0) \geq \varepsilon>0$, and $\underline{u}(x, 0) \leq \bar{u}(x, 0)$ in $\bar{\Omega}$. Then we have $\underline{u}(x, t) \leq \bar{u}(x, t)$ in $\bar{\Omega}_{T}$.

Proof Since $\underline{u}$ and $\bar{u}$ are lower and upper solutions of problem (1)-(3), we have

$$
\begin{aligned}
& \iint_{Q_{T}}\left(\underline{u}_{t}-\bar{u}_{t}\right) \phi d x d t+\iint_{Q_{T}}\left(|\nabla \underline{u}|^{p-2} \nabla \underline{u}-|\nabla \bar{u}|^{p-2} \nabla \bar{u}\right) \cdot \nabla \phi d x d t \\
& \quad \leq \iint_{Q_{T}} \phi\left[\left(\underline{u}^{m}-\bar{u}^{m}\right) \int_{\Omega} \underline{u}^{n} d y+\bar{u}^{m} \int_{\Omega}\left(\underline{u}^{n}-\bar{u}^{n}\right) d y-\alpha\left(\underline{u}^{r}-\bar{u}^{r}\right)\right] d x d t .
\end{aligned}
$$

Choosing a test function $\phi=(\underline{u}-\bar{u})_{+}=\max \{\underline{u}-\bar{u}, 0\}$, we get

$$
\begin{aligned}
& \iint_{Q_{T}}(\underline{u}-\bar{u})_{t}(\underline{u}-\bar{u})_{+} d x d t+\iint_{Q_{T}}\left(|\nabla \underline{u}|^{p-2} \nabla \underline{u}-|\nabla \bar{u}|^{p-2} \nabla \bar{u}\right) \cdot \nabla(\underline{u}-\bar{u})_{+} d x d t \\
& \leq \iint_{Q_{T}}(\underline{u}-\bar{u})_{+}\left[\int_{\Omega}\left(\underline{u}^{m}-\bar{u}^{m}\right) d y-\alpha\left(\underline{u}^{r}-\bar{u}^{r}\right)\right] d x d t .
\end{aligned}
$$

It follows from Lemma 1 that

$$
\left(|\nabla \underline{u}|^{p-2} \nabla \underline{u}-|\nabla \bar{u}|^{p-2} \nabla \bar{u}\right) \cdot \nabla(\underline{u}-\bar{u})_{+} \geq \min \left\{0, c|\nabla(\underline{u}-\bar{u})|^{p}\right\} \quad \text { for } p>2,
$$

and hence, one can see that

$$
\begin{aligned}
& \iint_{Q_{T}}(\underline{u}-\bar{u})_{t}(\underline{u}-\bar{u})_{+} d x d t \\
& \quad \leq \iint_{Q_{T}}(\underline{u}-\bar{u})_{+}\left[\left(\underline{u}^{m}-\bar{u}^{m}\right) \int_{\Omega} \underline{u}^{n} d y+\bar{u}^{m} \int_{\Omega}\left(\underline{u}^{n}-\bar{u}^{n}\right) d y-\alpha\left(\underline{u}^{r}-\bar{u}^{r}\right)\right] d x d t \\
& \quad \leq \iint_{Q_{T}}\left[\Phi_{1}(x, t) \int_{\Omega} \underline{u}^{n} d y+\bar{u}^{m}|\Omega| \Phi_{2}(x, t)+\alpha \Phi_{3}(x, t)\right](\underline{u}-\bar{u})_{+}^{2} d x d t,
\end{aligned}
$$

where

$$
\begin{aligned}
& \Phi_{1}(x, t)=m \int_{0}^{1}(\theta \underline{u}+(1-\theta) \bar{u})^{m-1} d \theta, \\
& \Phi_{2}(x, t)=n \int_{0}^{1}(\theta \underline{u}+(1-\theta) \bar{u})^{n-1} d \theta, \\
& \Phi_{3}(x, t)=r \int_{0}^{1}(\theta \underline{u}+(1-\theta) \bar{u})^{r-1} d \theta .
\end{aligned}
$$

Noticing that $\underline{u}(x, t)$ and $\bar{u}(x, t)$ are bounded functions, it follows from $r \geq 1, m \geq 1$, and $n \geq 1$ that $\Phi_{i}(i=1,2,3)$ are bounded nonnegative functions. If $0 \leq m<1$ or $0<n<1$, we have $\Phi_{2} \leq \varepsilon^{m-1}$ and $\Phi_{4} \leq \varepsilon^{n-1}$, since $\underline{u}(x, 0) \geq 0$ or $\bar{u}(x, 0) \geq \varepsilon>0$. Moreover, because $\underline{u}(x, 0) \leq \bar{u}(x, 0)$, we get

$$
\int_{\Omega}(\underline{u}-\bar{u})_{+}^{2} d x \leq C \iint_{Q_{T}}(\underline{u}-\bar{u})_{+}^{2} d x d t
$$


It follows from Gronwall's inequality that $(\underline{u}-\bar{u})_{+}=0$, and hence, $\underline{u}(x, t) \leq \bar{u}(x, t)$ in $\Omega \times(0, T)$.

For $x \in \partial \Omega$ and $y \in \Omega$, we have

$$
\underline{u}-\bar{u} \leq \int_{\Omega} \varphi(x, y)(\underline{u}-\bar{u}) d y .
$$

It is obvious that $\underline{u} \leq \bar{u}$ on $S_{T}$ if $\underline{u} \leq \bar{u}$ in $\Omega$. Therefore, we obtain $\underline{u}(x, t) \leq \bar{u}(x, t)$ in $\bar{\Omega}_{T}$.

Next, we state the local existence and uniqueness theorem without proof.

Theorem (Local existence and uniqueness) Suppose that $u_{0}(x) \in C(\bar{\Omega}) \cap W_{0}^{1, p}(\Omega)$. Then there exists $T_{0}>0$ such that problem (1.1)-(1.3) has a unique nonnegative solution $u \in$ $C\left(0, T ; L^{\infty}(\Omega)\right) \cap L^{p}\left(0, T ; W_{0}^{1, p}(\Omega)\right)$ for all $T<T_{0}$. Furthermore, either $T_{0}=\infty$ or

$$
\lim _{t \rightarrow T_{0}^{-}} \sup \|u(x, t)\|_{\infty}=\infty
$$

Remark 5 The local existence, in time, of nonnegative solutions of problem (1.1)-(1.3) can be obtained by using the fixed point theorem or the parabolic regular theory to get a suitable estimate in the standard limiting process (see $[18,19])$. We can obtain the uniqueness of solution to the problem by the comparison principle stated in Proposition 1 . The proof is more or less standard, and so it is omitted here.

\section{Proofs of main results}

In this section, we give detailed proofs of our main results to problem (1.1)-(1.3) by using the comparison principle and constructing suitable upper and lower solutions.

Proof of Theorem 1 Consider the following problem:

$$
v^{\prime}(t)=|\Omega| v^{m+n}-\alpha v^{r}, \quad v(0)=v_{0} .
$$

Since $m+n>r$, we know that $v^{m+n}+1>v^{r}$ and $|\Omega| v^{m+n}-\alpha v^{r} \geq(|\Omega|-\alpha) v^{m+n}-\alpha$, and hence, the solution of (3.1) is an upper solution of the following problem:

$$
v^{\prime}(t)=(|\Omega|-\alpha) v^{m+n}-\alpha, \quad v(0)=v_{0} .
$$

When $|\Omega|>\alpha$ and $m+n>1$, it is known that the solution of this problem blows up in finite time if $v_{0}>\left(\frac{\alpha}{|\Omega|-\alpha}\right) \frac{1}{m+n}$.

It is obvious that the solution of problem (3.1) is a lower solution of problem (1.1)-(1.3), when $\int_{\Omega} \varphi(x, y) d y \geq 1$ and $u_{0}(x)>v_{0}$. Therefore, the solution $u(x, t)$ blows up in finite time by Proposition 1 .

Proof of Theorem 2 Assume that $r>p-1$ and set $\bar{u}(x, t)=A>0$. For all $x \in \Omega$, choosing $A \geq \max \left\{\max _{\bar{\Omega}} u_{0}(x),\left(\frac{|\Omega|}{\alpha}\right)^{\frac{1}{r-m-n}}\right\}$, we get

$$
\bar{u}_{t}-\operatorname{div}\left(|\nabla \bar{u}|^{p-2} \nabla \bar{u}\right)-\bar{u}^{m} \int_{\Omega} \bar{u}^{n} d x+\alpha \bar{u}^{r}=\alpha A^{r}-|\Omega| A^{m+n} \geq 0 .
$$


For all $x \in \partial \Omega$, we have

$$
\bar{u}(x, t)=A \geq \int_{\Omega} A \varphi(x, y) d y=\int_{\Omega} \varphi(x, y) \bar{u} d y .
$$

By Proposition 1, we can obtain the result.

(ii) Assume that $r<p-1$ and let $\widetilde{\Omega}$ be a bounded domain in $R^{N}$ such that $\Omega \subset \widetilde{\Omega}$. If $\psi(x)$ solves the following problem:

$$
\begin{aligned}
& -\operatorname{div}\left(|\nabla \psi|^{p-2} \nabla \psi\right)=1, \quad x \in \widetilde{\Omega}, \\
& \psi(x)=0, \quad x \in \partial \widetilde{\Omega},
\end{aligned}
$$

one can easily see that

$$
\frac{\sup _{\widetilde{\Omega}} \psi}{\inf _{\bar{\Omega}} \psi}<a \text { for some } a>1
$$

Taking $\sup _{\widetilde{\Omega}} \psi=a \varepsilon$, where $\varepsilon>0$, it follows from (3.4) that

$$
\inf _{\bar{\Omega}} \psi>\varepsilon \quad \text { and } \quad \sup _{\bar{\Omega}} \psi<a \varepsilon
$$

Setting $\bar{u}(x, t)=A \psi(x)$, where $A \geq \max \left\{\max _{\bar{\Omega}} u_{0}(x),\left[(\alpha \varepsilon)^{m+n}|\Omega|\right]^{\frac{1}{p-1-m-n}}\right\}$, we have

$$
\bar{u}_{t}-\operatorname{div}\left(|\nabla \bar{u}|^{p-2} \nabla \bar{u}\right)-\bar{u}^{m} \int_{\Omega} \bar{u}^{n} d x+\alpha \bar{u}^{r} \geq 0 .
$$

For all $x \in \partial \Omega$,

$$
\bar{u}(x, t)=A \psi(x) \geq A \int_{\Omega} \varphi(x, y) a \varepsilon d y \geq \int_{\Omega} \varphi(x, y) A \psi(y) d y=\int_{\Omega} \varphi(x, y) \bar{u}(y, t) d y .
$$

This completes the proof.

Proof of Theorem 3 We first show the existence of a global solution.

(i) Assume that $r>p-1$ and set $\bar{u}(x, t)=A$. Choosing a constant $A$ such that $\max _{\bar{\Omega}} u_{0}(x) \leq$ $A \leq\left(\frac{\alpha}{|\Omega|}\right)^{\frac{1}{m+n-r}}$, one can deduce the desired result by using a similar argument as the one used for (i) of Theorem 1 .

(ii) Assume that $r<p-1$ and set $\bar{u}(x, t)=A \psi(x)$, where $\psi(x)$ is the function defined in (3.2)-(3.4). Choosing a constant $A$ such that $\max _{\bar{\Omega}} u_{0} \varepsilon^{-1} \leq A \leq\left[(a \varepsilon)^{m+n}|\Omega|\right]^{\frac{1}{m+n-p+1}}$ and using the same argument as the one used for (ii) of Theorem 1 , one can see that there exists a global solution of problem (1.1)-(1.3).

Secondly, we establish a self-similar blow-up solution in order to show a blow-up of the solution. We now assume that $\omega(x) \in C^{1}(\bar{\Omega}), \omega(x) \geq 0, \omega(x)$ is not identically zero, and $\left.\omega(x)\right|_{\partial \Omega}=0$. Without loss of generality, we assume that $0 \in \Omega$ and $\omega(0)>0$.

Let $\underline{u}(x, t)=(T-t)^{-\gamma} V(\xi), V(\xi)=1-\xi^{2}$, and let $\xi=|x|(T-t)^{-\mu}$, where $0<T<1$ and $\gamma, \mu>0$. We know that

$$
\operatorname{supp} \underline{u}_{+}(\cdot, t)=\overline{B\left(0, R(T-t)^{\mu}\right)} \subset \overline{B\left(0, R T^{\mu}\right)} \subset \Omega \text {, }
$$


for sufficiently small $T>0$. Calculating the derivative of $\underline{u}$, we obtain the equations

$$
\begin{aligned}
& \underline{u}_{t}=\frac{\gamma V(\xi)}{(T-t)^{\gamma+1}}+\frac{\mu V^{\prime}(\xi)|x|}{(T-t)^{\gamma}(T-t)^{\mu+1}}=\frac{\gamma V(\xi)+\mu \xi V^{\prime}(\xi)}{(T-t)^{\gamma+1}}, \\
& \begin{aligned}
& \nabla \underline{u}=\nabla\left[(T-t)^{-\gamma}\left(1-\xi^{2}\right)\right]=(T-t)^{-\gamma}(-2 \xi) \cdot \nabla \xi=(T-t)^{-\gamma-2 \mu}(-2 x), \\
& \Delta \underline{u}=\frac{2 N}{(T-t)^{\gamma+2 \mu}}, \\
& \operatorname{div}\left(|\nabla \underline{u}|^{p-2} \nabla \underline{u}\right)=|\nabla \underline{u}|^{p-2} \triangle \underline{u}+(p-2)|\nabla \underline{u}|^{p-4}(\nabla \underline{u})^{\prime}\left(H_{x}(\underline{u})\right) \cdot \nabla \underline{u} \\
&=|\nabla \underline{u}|^{p-2} \triangle \underline{u}+(p-2)|\nabla \underline{u}|^{p-4} \sum_{j=1}^{N} \sum_{i=1}^{N} \frac{\partial \underline{u}}{\partial x_{i}} \frac{\partial \underline{u}}{\partial x_{i} \partial x_{j}} \frac{\partial \underline{u}}{\partial x_{j}},
\end{aligned}
\end{aligned}
$$

where $H_{(x)}(\underline{u})$ is the Hessian matrix of $\underline{u}$. Let $d(\Omega)=\operatorname{diam}(\Omega)$. We then have

$$
\begin{aligned}
& \left|\operatorname{div}\left(|\nabla \underline{u}|^{p-2} \nabla \underline{u}\right)\right| \leq \frac{2 N(p-1)(d(\Omega))^{p-2}}{(T-t)^{(\gamma+2 \mu)(p-1)}}, \\
& \underline{u}^{m} \int_{\Omega} \underline{u}^{n}(y, t) d y=\frac{V}{(T-t)^{(m+n) \gamma}} \int_{B\left(0, R(T-t)^{\nu}\right)} V^{n}\left(\frac{|x|}{(T-t)^{\nu}}\right) d x=\frac{M}{(T-t)^{(m+n) \gamma-N \mu}},
\end{aligned}
$$

where $M=V^{m} \int_{B(0, R)} V^{n}(|\xi|) d \xi>0$. Then we get the inequalities

$$
\begin{aligned}
\underline{u}_{t} & -\operatorname{div}\left(|\nabla \underline{u}|^{p-2} \nabla \underline{u}\right)-\underline{u}^{m} \int_{\Omega} \underline{u}^{n}(y, t) d y+\alpha u^{r} \\
& \leq \frac{\gamma V(\xi)+\mu \xi V^{\prime}(\xi)}{(T-t)^{\gamma+1}}+\frac{2 N(p-1)(d(\Omega))^{p-2}}{(T-t)^{(\gamma+2 \mu)(p-1)}}-\frac{M}{(T-t)^{(m+n) \gamma-N \mu}}+\frac{\alpha V^{r}}{(T-t)^{r \gamma}} \\
& \leq \frac{\gamma}{(T-t)^{\gamma+1}}+\frac{2 N(p-1)(d(\Omega))^{p-2}}{(T-t)^{(\gamma+2 \mu)(p-1)}}+\frac{\alpha}{(T-t)^{r \gamma}}-\frac{M}{(T-t)^{(m+n) \gamma-N \mu}} .
\end{aligned}
$$

Since $m+n>\max \{p-1, r\}$, we can choose $\gamma$ such that $\max \{p-1, r\}<\frac{1+\gamma}{\gamma}<m+n$ and $\mu$ sufficiently small such that $\mu<\min \left\{\frac{(m+n-1) \gamma-1}{N}, \frac{(m+n-r) \gamma}{N}, \frac{(m+n-p+1) \gamma}{N+2(p-1)}\right\}$. We then obtain the inequality

$$
(m+n) \gamma-N \mu>\max \{\gamma+1, r \gamma,(\gamma+2 \mu)(p-1)\} .
$$

By (3.6) and (3.7), we know that

$$
\underline{u}_{t}-\operatorname{div}\left(|\nabla \underline{u}|^{p-2} \nabla \underline{u}\right)-\underline{u}^{m} \int_{\Omega} \underline{u}^{n}(y, t) d y+\alpha u^{r} \leq 0 \quad \text { in } \bar{\Omega}_{T}
$$

for sufficiently small $T>0$.

If $x \in \partial \Omega, \omega(0)>0$, and $\omega$ is continuous, it is known that there exist positive constants $\varepsilon$ and $\rho$ such that $\omega \geq \varepsilon$ for $x \in B(0, \rho)$. We then have $B\left(0, R T^{\sigma}\right) \subset B(0, \rho) \subset \Omega$ if $T$ is small enough, and so $\underline{u} \leq \int_{\Omega} \varphi(x, y) \underline{u} d y$ on $S_{T}$. It follows from (3.4) that $\underline{u}(x, 0) \leq K_{0} \omega(x)$ for sufficiently large $K_{0}$. Therefore, one can see that the solution of problem (1.1)-(1.3) exists no later than $t=T$ provided that $u_{0} \geq K_{0} \omega(x)$. This implies that the solution blows up in finite time for large enough initial data. 
Proof of Theorem 4 (i) If $r>p-1$, choosing $A$ large enough so that $A \geq \max _{\bar{\Omega}} u_{0}(x)$, one can see that $\bar{u}(x, t)=A$ is the upper solution of problem (1.1)-(1.3), and hence, the result follows.

To show the blow-up result, we only need to establish a blow-up of a suitable lower solution. Let $v(t)$ be a solution of the following equation:

$$
\begin{aligned}
& v^{\prime}(t)=c_{1} v^{\beta_{1}}-c_{2} v^{\beta_{2}}, \quad t>0, \\
& v(0)=v_{0}>0,
\end{aligned}
$$

where $c_{1}=\frac{\psi^{m} \int_{\Omega} \psi^{n} d x-\alpha \psi^{r}}{l_{1} \psi}, c_{2}=\frac{1}{l_{1} \psi}, l_{1}>1, \beta_{1}=(r-1) l_{1}+1$, and $\beta_{2}=(p-2) l_{1}+1$. By the assumptions of Theorem 4 , we know that $c_{1}>0$ and $\beta_{1}>\beta_{2}$. It is obvious that there exists a constant $T^{*}$ such that

$$
0<T^{*}<+\infty, \quad \lim _{t \rightarrow T^{*}} v(t)=+\infty .
$$

Set $\underline{u}(x, t)=v^{l_{1}}(t) \psi(x)$, where $\psi(x)$ is the function defined in (3.2)-(3.4). For all $(x, t) \in$ $Q_{T}$, a series of computations show that

$$
\begin{aligned}
\underline{u}_{t} & -\operatorname{div}\left(|\nabla \underline{u}|^{p-2} \nabla \underline{u}\right)-\underline{u}^{m} \int_{\Omega} \underline{u}^{n}(y, t) d y+\alpha \underline{u}^{r} \\
& =l_{1} \psi v^{l_{1}-1} v^{\prime}+v^{l_{1}(p-1)}-v^{(m+n) l_{1}} \psi^{m} \int_{\Omega} \psi^{n} d x+\alpha v^{r l_{1}} \psi^{r} \\
& =l_{1} \psi v^{l_{1}-1}\left(v^{\prime}+\frac{1}{l_{1} \psi} v^{(p-2) l_{1}+1}-\frac{\psi^{m} \int_{\Omega} \psi^{n} d x-\alpha \psi \psi^{r}}{l_{1} \psi} v^{l_{1}(r-1)+1}\right) \\
& \leq 0 .
\end{aligned}
$$

For all $(x, t) \in S_{T}$, we also have

$$
\underline{u}(x, t)=v^{l_{1}} \psi(x)<v^{l_{1}} a \varepsilon \leq \int_{\Omega} \varphi(x, y) v^{l_{1}} \psi(x) d y=\int_{\Omega} \varphi(x, y) \underline{u}(y, t) d y .
$$

(ii) If $p-1>r$, we have $m+n=p-1>r$. Let $\bar{u}(x, t)=A \psi(x)$. By the assumptions of Theorem 4, we know that

$$
\begin{aligned}
\bar{u}_{t} & -\operatorname{div}\left(|\nabla \bar{u}|^{p-2} \nabla \bar{u}\right)-\bar{u}^{m} \int_{\Omega} \bar{u}^{n} d x+\alpha \bar{u}^{r} \\
& =A^{p-1}-A^{m+n} \psi^{m} \int_{\Omega} \psi^{n} d x+\alpha A^{r} \varphi^{r} \\
& =A^{m+n}\left(1-\psi^{m} \int_{\Omega} \psi^{n} d x\right)+\alpha A^{r} \varphi^{r} \geq 0 .
\end{aligned}
$$

For all $(x, t) \in S_{T}$, we get

$$
\bar{u}(x, t)=A \psi(x)>A \frac{a \varepsilon}{a} \geq \int_{\Omega} \varphi(x, y) A \psi(y) d y=\int_{\Omega} \varphi(x, y) \bar{u}(y, t) d y .
$$

Choosing $u_{0}$ such that $u_{0}(x)<A \psi(x)<A a \varepsilon$, one can see that there exists a global solution of problem (1.1)-(1.3). 
For the blow-up result, we again consider the following problem:

$$
\begin{aligned}
& v^{\prime}(t)=c_{1} v^{\beta_{1}}-c_{2} v^{\beta_{2}}, \quad t>0, \\
& v(0)=v_{0}>0,
\end{aligned}
$$

where $c_{1}=\psi^{m} \int_{\Omega} \psi^{n} d x-1>0, c_{2}=\frac{\alpha \psi^{r-1}}{l_{1}}, \beta_{1}=(p-2) l_{1}+1, \beta_{2}=(r-1) l_{1}+1$, and $l_{1}>1$. It is obvious that $\beta_{1}>\beta_{2}$, since $p-1>r$. Setting $\underline{u}(x, t)=v^{l_{1}}(t) \psi(x)$, the result follows by the same argument as the one for the case $r>p-1$.

\section{Competing interests}

The authors declare that they have no competing interests.

\section{Authors' contributions}

All authors contributed equally to the manuscript and read and approved the final manuscript.

\section{Acknowledgements}

This work is supported by the Natural Science Foundation of Shandong Province of China (ZR2012AM018). The authors would like to deeply thank all the reviewers for their insightful and constructive comments.

Received: 24 November 2012 Accepted: 30 May 2013 Published: 27 June 2013

\section{References}

1. Bebernes, J, Eberly, D: Mathematical Problems from Combustion Theory. Springer, New York (1989)

2. Pao, CV: Nonlinear Parabolic and Elliptic Equations. Plenum, New York (1992)

3. Furter, J, Grinfield, M: Local vs. non-local interactions in populations dynamics. J. Math. Biol. 27, 65-80 (1989)

4. Calsina, A, Perello, C, Saldana, J: Non-local reaction-diffusion equations modelling predator-prey coevolution. Publ. Mat. 32, 315-325 (1994)

5. Allegretto, W, Fragnelli, G, Nistri, P, Papin, D: Coexistence and optimal control problems for a degenerate predator-prey model. J. Math. Anal. Appl. 378, 528-540 (2011)

6. Day, WA: Extensions of property of heat equation to linear thermoelasticity and other theories. Q. Appl. Math. 40, 319-330 (1982)

7. Day, WA: A decreasing property of solutions of parabolic equations with applications to thermoelasticity. Q. Appl. Math. 40, 468-475 (1983)

8. Friedman, A: Monotonic decay of solutions of parabolic equations with nonlocal boundary conditions. Q. Appl. Math. 44(3), 401-407 (1986)

9. Pao, CV: Asymptotic behavior of solutions of reaction-diffusion equations with nonlocal boundary conditions. J. Comput. Appl. Math. 88(1), 225-238 (1998)

10. Wang, YL, Mu, CL, Xiang, ZY: Blow-up of solutions to a porous medium equation with nonlocal boundary condition. Appl. Math. Comput. 192, 579-585 (2007)

11. Lin, ZG, Liu, YR: Uniform blow-up profiles for diffusion equations with nonlocal source and nonlocal boundary. Acta. Math. Sci. 24, 443-450 (2004)

12. Cui, ZJ, Yang, ZD: Roles of weight functions to a nonlinear porous medium equation with nonlocal source and nonlocal boundary condition. J. Math. Anal. Appl. 342, 559-570 (2008)

13. Souplet, P: Blow-up in nonlocal reaction-diffusion equations. SIAM J. Math. Anal. 29(6), 1301-1334 (1998)

14. Li, FC, Xie, CH: Global and blow-up solutions to a $p$-Laplace equation with nonlocal source. Comput. Math. Appl. 46 , 1525-1533 (2003)

15. Wang, YL, Mu, CL, Xiang, ZY: Properties of positive solution for nonlocal reaction diffusion equation with nonlocal boundary. Bound. Value Probl. 2007, Article ID 064579 (2007). doi:10.1155/2007/64579

16. Wang, MX, Wang, Y: Properties of positive solutions for non-local reaction-diffusion problems. Math. Methods Appl. Sci. 19, 1141-1156 (1996)

17. Diaz, Jl: Nonlinear Partial Differential Equations and Free Boundaries: Elliptic Equations, vol. I. Pitman, London (1985)

18. Dibenedetto, E: Degenerate Parabolic Equations. Springer, New York (1993)

19. Li, FC: Global existence and blow-up of solutions to a nonlocal quasilinear degenerate parabolic system. Nonlinear Anal. 67, 1387-1402 (2007)

doi:10.1186/1029-242X-2013-301

Cite this article as: Fang and Zhang: Influence of weight functions to a nonlocal $p$-Laplacian evolution equation with inner absorption and nonlocal boundary condition. Journal of Inequalities and Applications 2013 2013:301. 